\title{
La Guerre de Séparation catalane (1640) au ralenti
}

Note de lecture critique

\section{Mathias Ledroit}

\section{OpenEdition}

Journals

Electronic version

URL: http://journals.openedition.org/agedor/1853

DOI: 10.4000/agedor.1853

ISSN: 2104-3353

\section{Publisher}

Laboratoire LISAA

\section{Electronic reference}

Mathias Ledroit, « La Guerre de Séparation catalane (1640) au ralenti », L'Âge d'or [Online], 10 | 2017, Online since, connection on 10 December 2020. URL : http://journals.openedition.org/agedor/1853 ; DOI : https://doi.org/10.4000/agedor.1853

This text was automatically generated on 10 December 2020.

L'Âge d'or. Images dans le monde ibérique et ibéricoaméricain 


\section{La Guerre de Séparation catalane (1640) au ralenti}

Note de lecture critique

Mathias Ledroit

\section{REFERENCES}

Barcelone, Rafael Dalmau Editor

\section{Les auteurs et leur propos}

Dans Ambaixadors catalans a Madrid. Els inicis de la Guerra de Separació (1640-1641), Antoni Muñoz et Josep Catà entreprennent d'étudier la mission que les ambassadeurs de Barcelone et de la Generalitat ont menée auprès de Philippe IV, entre 1640 et 1641 . Ils replacent cette légation dans le contexte des soulèvements populaires survenus en Catalogne contre les soldats des tercios. Ils entendent ainsi mettre en lumière la façon dont la révolte populaire (mieux connue dans l'historiographie sous le nom de "Guerra dels Segadors") s'est progressivement politisée au point d'entraîner, en janvier 1641, la proclamation de la République catalane puis l'acclamation de Louis XIII comme Comte de Barcelone. À partir de documents d'époque souvent inédits, recueillis à l'Archivo General de Simancas, dans l'Archivo Nacional de Madrid et à la Biblioteca Nacional de España, les auteurs s'attachent à reconstituer la narration des événements en optant pour le temps court (p. 8). Ils proposent ainsi de faire un ralenti ${ }^{1}$, dont le but est d'offrir un " discours alternatif », c'est-à-dire de " considérer que d'autres options, différentes de celles survenues selon le récit officiel, étaient possibles, bien qu'elles ne se soient pas imposées à l'époque » (p. 8). Les deux auteurs font ici référence aux travaux menés dans les années 1960-1970 par John H. Elliott, Josep Sanabre et Eulogio Zudaire, dont les ouvrages, pourtant considérés comme «centralistes", font encore autorité aujourd'hui². 
2 Il importe de préciser d'emblée qu'Antoni Muñoz et Josep Catà sont tous deux membres du Cercle Català d'Història. Cette association d'historiens professionnels et amateurs promeut la récupération et la diffusion de la mémoire catalane et, simultanément, condamne «tous les épisodes de violences, de persécution, d'arrestation de répression de l'État espagnol contre la Catalogne et contre les citoyens et ses citoyennes $»^{3}$. C'est donc dans cette perspective que les auteurs énoncent, dès l'introduction, leur objectif de « montrer la valeur et la préparation [des ambassadeurs], afin de les honorer comme il se doit $»^{4}$. L'approche se veut donc volontairement orientée et partisane - «non neutre ", pour reprendre l'expression même des auteurs (p. 8). Antoni Muñoz et Josep Catà posent en effet dès l'introduction la question de l'engagement de l'historien. Ils estiment qu'un historien n'est jamais neutre : tant sa démarche que sa méthodologie et sa sélection de documents d'archives sont guidées par des convictions personnelles (p. 7). Le livre s'ouvre sur un chapitre synthétique dans lequel Antoni Muñoz et Josep Catà offrent une sélection d'affrontements politiques survenus entre les institutions catalanes - le Conseil des Cent de Barcelone et la Generalitat - et le gouvernement royal entre 1621 et 1640. Il s'agit pour les auteurs d'introduire le «biais » qui éclairera le reste de leur étude, c'est-à-dire : montrer comment, depuis 1621, le gouvernement royal a cherché à réduire les Catalans à l'obéissance (p.11) par la mise en place, dès 1635, d'une politique répressive calculée (p. 16). L'introduction de ce «biais » interpelle en ceci qu'il nous plonge de plain-pied dans la relation intriquée et indécise qu'entretiennent histoire et mémoire. Ils soulèvent en effet de multiples questions d'ordre épistémologique, herméneutique mais aussi politique sur lesquelles il sera important de revenir plus avant.

\section{Grandes lignes de l'argumentation de l'ouvrage}

3 L'argumentation de l'ouvrage peut être résumée en six points principaux. 1) En 1640, alors que les affrontements se multipliaient en Catalogne entre les soldats et les populations locales, le gouvernement royal de la Monarchie Catholique n'a pas cherché à mettre un terme aux excès des soldats, choisissant plutôt de faire la sourde oreille aux suppliques des ambassadeurs, qui avaient été mandatés par les institutions catalanes pour obtenir le retrait des soldats et le châtiment de leurs excès (p. 21-26). Au contraire, la politique des logements dits à la Lombarde aurait été une stratégie mise en œuvre par la Junta de Ejecución, chapeautée par le Comte-Duc, pour occuper la Catalogne et pour la soumettre à l'absolutisme castillan (p.60). Le Comte-Duc et Jerónimo Villanueva, protonotaire pour la Couronne d'Aragon, auraient encouragé une « escalade d'action-réaction» (p. 43), afin que les affrontements entre les soldats et les populations locales se multiplient et puissent, in fine, justifier l'invasion militaire de la Catalogne et l'abolition des «libertés catalanes». C'est dans cette dynamique qu'il faudrait interpréter l'emprisonnement du député catalan du braç militar Francesc Tamarit ordonné par le gouvernement royal en mars 1640 (p.26), mais aussi la tentative avortée de Philippe IV de convoquer les Cortès en 1640. Selon les promoteurs de la Generalitat, ces dernières auraient été, pour le roi, une occasion camouflée d'entrer en Catalogne à la tête d'une armée d'occupation (p.148). 2) Les émeutes survenues à Barcelone le 22 mai et le 7 juin 1640 n'auraient rien de spontané et résulteraient, au contraire, d'une résistance paysanne organisée autour de l'institution d'origine médiévale du somatén. Elles auraient eu pour objectif d'organiser la libération du 
député Tamarit le 22 mai 1640 et de châtier, par la mort, les représentants de l'autorité royale, jugés sinon instigateurs, du moins complices des exactions des tercios. À ce propos, Antoni Muñoz et Josep Catà avancent l'hypothèse selon laquelle l'attaque contre le vice-roi Santa Coloma aurait initialement été planifiée pour le 22 mai. Elle aurait été repoussée à dessein au 7 juin afin qu'elle puisse coïncider avec les solennités du Corpus Christi et l'arrivée des moissonneurs à Barcelone (chapitre vII). 3) Le Comte de Santa Coloma n'aurait pas été assassiné, mais il serait mort dans un accident advenu alors qu'il tentait de fuir les moissonneurs insurgés (chapitre VII). 4) Antoni Muñoz et Josep Catà consacrent un chapitre à émettre des hypothèses sur l'identité du mystérieux "capitaine de l'armée chrétienne » (" el capità de l'Exèrcit cristià ») et suggèrent ainsi que la forte dimension religieuse qui accompagne les revendications des paysans catalans ne serait dans le fond qu'un «facteur agglutinant " et donc secondaire qui serait venu s'agréger à la dimension patriotique du mouvement armé (chapitre VIII). 5) La République catalane (16 janvier au 23 janvier 1641) n'aurait pas été une simple transition destinée à faciliter le changement d'obédience entre Philippe IV et Louis XIII comme l'avait suggéré, en son temps, John H. Elliott. Au contraire, elle aurait été une tentative frustrée de faire de la Catalogne un État indépendant semblable au modèle de la République de Venise et répondrait, par conséquent, à des aspirations indépendantistes déjà bien ancrées dans les esprits des Catalans du XVII siècle (p. 127-128). Pau Claris aurait dès lors été le tout premier président républicain des Catalans, ce qui lui aurait valu d'être empoisonné par des agents de Louis XIII et de Richelieu, lesquels le jugeaient trop gênant ${ }^{5}$. 6) En s'appuyant sur la correspondance échangée entre les ambassadeurs et leurs mandataires ainsi que sur les memoriales qu'ils ont remis au Comte-Duc, Antoni Muñoz et Josep Catà montrent comment l'explosion de violence survenue en Catalogne à la suite de la récupération de la place forte de Salses a fragilisé la position des émissaires et frustré leur tentative de négocier la libération du député Tamarit, l'évacuation des tercios et le châtiment exemplaire contre les excès perpétrés par les soldats, notamment les sacrilèges (p. 107). Ce faisant, les deux auteurs confirment, à la lumière de la documentation "confisquée " par le gouvernement royal à Valencas, ambassadeur de la Generalitat pour le braç reial (p. 133 et suivantes), des thèses déjà avancées par d'autres travaux sur le sujet, lesquels n'apparaissent pourtant pas dans les références bibliographiques de l'ouvrage ${ }^{6}$. Mais le but recherché par les deux historiens porte davantage, semble-t-il, sur l'hostilité dont les ambassadeurs ont été victimes à Madrid. Il est en effet question, dans l'ouvrage, des humiliations et des vexations infligées aux ambassadeurs par le Comte-Duc et Jerónimo Villanueva d'une part et du désintérêt de Philippe IV pour leurs suppliques d'autre part. La narration des faits sur le temps court de la légation permet ainsi de mettre en lumière la façon dont les ambassadeurs sont progressivement devenus les otages du gouvernement royal qui, en les assignant à résidence (en les " séquestrant », p. 155) en a fait une monnaie d'échange dans le but de fragiliser l'alliance naissante entre la Catalogne et la France et de temporiser afin de parachever les préparatifs de l'invasion militaire de la Principauté, avant de les échanger contre les fils de la duchesse de Cardona, qui avait été désignée pour mener une ultime tentative de réconciliation, perdue d'avance selon les auteurs, entre les institutions catalanes et le gouvernement. Par ailleurs, Antoni Muñoz et Josep Catà estiment que le père capucin, Bernardí de Manlleu, envoyé extraordinaire de la Generalitat, Pau Claris, était, en réalité, un espion retourné, à la solde d'Olivares (p. 158). L'affirmation nécessiterait, ici, d'être appuyée pour deux raisons. D'une part, parce que les auteurs s'appuient, sans citer de document 
en note, sur une rumeur. D'autre part, parce qu'ils n'incluent pas, dans leur analyse, la correspondance échangée tout au long de l'ambassade entre Bernardí de Manlleu et son mandataire, laquelle est pourtant conservée dans les archives de la Biblioteca de Catalunya.

Pour ce qui concerne l'objet central de l'ouvrage, à savoir celui des ambassadeurs, sans doute aurait-il mérité d'être inscrit dans la durée. Car il s'agit d'un mécanisme institutionnel largement attesté par la documentation de l'époque, mais sur lequel la discipline historique ne s'est jamais encore penchée. Certaines problématiques posées par les auteurs auraient ainsi pu être creusées, comme par exemple celle du ou des modes de négociation, celle du statut des sources et celle, enfin, de l'efficacité de ce mécanisme auquel Barcelone et la Generalitat de Catalogne ont souvent eu recours dans le but de négocier directement avec le roi. À titre d'exemple, on compte, pour le seul règne de Philippe IV, une ambassade par an envoyée à Madrid et il arrivait même, comme cela fut le cas en 1632, que deux ambassadeurs de la ville se trouvent à Madrid en même temps. Une autre question qu'il aurait été utile de traiter concerne le statut des ambassadeurs, notamment lorsque surgit la question de leur emprisonnement. Certaines réactions d'Olivares face aux ambassadeurs conduisent Antoni Muñoz et Josep Catà à conclure que les émissaires des institutions catalanes n'étaient pas traités comme des "sujets politiques", autrement dit qu'ils ne recevaient pas le traitement conforme à leur fonction. Or, il est pour l'heure impossible de cerner le statut des ambassadeurs catalans envoyés à Madrid à l'époque moderne. Et qui plus est, leur statut même soulève un vif débat dans les années $1630^{7}$. La ville de Barcelone et la Generalitat de Catalogne sont, en effet, les deux seules institutions disposant du privilège d'envoyer des "ambassadeurs", tandis que les autres institutions catalanes envoient, elles, des «syndics ». Le problème est soulevé en 1632 lors de la légation de Francesc Bru, tel qu'on le lit dans la relation d'ambassade que celui-ci adresse au Conseil des Cent à son retour de Madrid'. Les ambassadeurs catalans ont-ils, comme cela est le cas pour le représentant d'un souverain, un statut similaire à celui de ses mandataires, à savoir les députés de la Generalitat et les Conseillers de Barcelone? Bénéficient-ils d'une immunité ou de traitements spécifiques, comme cela est le cas du président de la Generalitat qui, en tant qu'ecclésiastique, n'est pas soumis à la justice civile et ne peut, par conséquent, être emprisonné sur ordre de la justice royale? De même, est-il pertinent de parler d'une "violation» de la correspondance des ambassadeurs de la part du Comte-Duc (p.158), et si oui, dans quelle mesure? Il semblerait - mais encore conviendrait-il de le démontrer - que les ambassadeurs aient eu l'habitude d'entretenir, au cours de toutes leurs missions, une double correspondance avec leurs mandataires; l'une privée, à laquelle ils étaient les seuls à avoir accès, et l'autre publique, qui bien que leur étant destinée pouvait être consultée par le roi et le Comte-Duc. C'est du moins ce que suggèrent plusieurs relations d'ambassadeurs barcelonais, à l'instar de celle de Francesc Bru en 1632.

En réalité, l'ouvrage n'offre pas une étude proprement dite du mécanisme des ambassades. Les auteurs ont choisi d'étudier cette légation comme un prisme permettant de retracer la dégradation des rapports entre Madrid et Barcelone et la montée des tensions qui ont préludé à la rupture de 1641. La documentation inédite et de grande valeur sur laquelle ils s'appuient ne nous renseigne pas tant sur les ambassades que sur la dimension sociale et politique de la révolte des Catalans, du moins dans sa phase initiale. 
Bien que fortement contestée par les historiens, l'approche narrative des événements de 1640-1641 sur le temps court permet ici aux auteurs de faire un ralenti sur les affrontements entre les soldats des tercios et les populations locales (chapitres IV, VI, VII, x et XIII), et particulièrement dans l'Ampurdan entre janvier et juin 1640 (p.63-72) et dans les Comtés du Roussillon et du Conflent entre juin 1640 et janvier 1641 (p. 163-175). Par ce procédé, Antoni Muñoz et Josep Catà mettent en lumière la capacité de mobilisation des populations locales contre les excès des soldats - incendies d'églises, villes ou villages bombardés - et mettent ainsi en lumière l'origine sociale et la dimension apolitique des premiers soulèvements populaires (chapitre IV). Ce que cherchent les populations locales, c'est de se venger des abus commis par les soldats. La charge sociale de la révolte des Catalans de 1640 est encore un angle mort de la recherche, en partie parce que les sources sont trop rares pour écrire une histoire de ses origines socio-économiques ${ }^{9}$. Aussi la méthode narrative apparaît-elle ici sinon comme une fin, du moins comme un moyen d'avancer dans ce domaine. Appuyé sur une partie de la correspondance de Juan de Garay, maestre de campo general de l'armée de Philippe IV dans les Comtés, le chapitre XIII révèle la façon dont la violence exercée par les soldats des tercios à l'encontre des populations locales a été systématisée dans la seconde moitié de l'année 1640 (p.171). Les institutions catalanes et le gouvernement de la Monarchie ont été débordés par ces soulèvements qui ont provoqué l'effondrement de l'autorité royale dans un premier temps et, dans un second temps, celui des institutions catalanes (p. 263). Sur ce sujet, l'ouvrage encourage à aborder la révolte dans sa dimension sociale, en s'intéressant plus spécifiquement à l'entrée du peuple en politique. La description des soulèvements populaires à laquelle se livrent les auteurs laisse penser qu'il y aurait, en effet, une piste à suivre sur les pratiques sociales liées aux révolutions. On pourra, par exemple, envisager de relire, à la lumière des travaux effectués au sujet des pratiques sonores dans l'espace public, la signification des cris lancés tels que "Via fora!", intimement lié à l'institution du somatén, ou encore "Visca lo Rey! Muyran los traidors!" qu'on retrouve non seulement dans tous les mouvements d'insurrection en Catalogne entre le Moyen Âge et l'époque moderne ${ }^{10}$.

7 Le chapitre XIII montre en outre que les Comtés ont constitué un facteur important dans la montée des tensions entre les institutions catalanes et le gouvernement royal. Antoni Muñoz et Josep Catà confirment que le bombardement de Perpignan (15-16 juin 1640) et la tentative manquée de négociation menée par le vice-roi Cardona entre le gouvernement royal et les institutions catalanes dans les jours suivants ont eu un rôle déclencheur dans l'engagement du Conseil des Cent et de la Generalitat de Catalogne dans la guerre de résistance contre les armées royales (p. 123). Ils posent ainsi en creux la question de la place des Comtés dans la politique catalane de la Monarchie Catholique, mais aussi dans sa politique militaire contre la France. Les Catalans du XVII ${ }^{\mathrm{e}}$ siècle les considéraient, il est vrai, comme la «serrure » - el pany - de la Monarchie pour reprendre une expression souvent utilisée dans les textes de l'époque. Or, c'est là un thème encore trop peu traité par l'historiographie actuelle qui a plutôt fait porter ses efforts sur la difficile intégration des Comtés dans la Monarchie Française au lendemain du Traité des Pyrénées (1659) ${ }^{11}$. L'ouvrage nous invite donc à repenser sinon à penser plus simplement - la place stratégique de ces territoires désormais français dans la vie politique de la Catalogne antérieure au Traité des Pyrénées de 1659.

8 D'un point de vue méthodologique, l'approche des auteurs est ouvertement tendancieuse. Leur but est, en effet, de souligner l'oppression dont la Catalogne a été 
victime en 1640-1641. Ils s'inscrivent ainsi dans le discours victimisant qui caractérise l'essentiel de l'historiographie catalane des vingt dernières années. Trouvant leur inspiration dans l'essai publié par Eva Serra, trésorière du Cercle Català d'Història, à l'occasion du $350^{\mathrm{e}}$ anniversaire de la révolte de 1640 , ils assimilent le gouvernement royal de la Monarchie catholique à un gouvernement étranger qui, depuis l'union dynastique entre Ferdinand d'Aragon et Isabelle de Castille en 1469, a exercé une répression systématique contre la Catalogne ${ }^{12}$. D'où le fait que les deux auteurs se réfèrent à Philippe IV comme roi de Castille, aux soldats comme armée castillane, qu'ils parlent d'un gouvernement castillan pour désigner le gouvernement royal dans son opposition au gouvernement catalan. Ils maintiennent, en outre, une constante ambiguïté quant à l'indépendance - que la Catalogne aurait perdue en 1714, ce qui reviendrait à dire qu'elle était indépendante en 1640 - et quant à l'opposition entre l'État castillan et le Gouvernement catalan (note 14 de la p. 15).

9 L'ouvrage se situe dans le domaine de la mémoire et, serions-nous tentés de dire même, à cheval entre la «mémoire manipulée » et la « mémoire obligée " pour reprendre les termes de l'analyse de Paul Ricœur ${ }^{13}$. Le projet des auteurs correspond à l'esprit du Cercle Català d'Història, c'est-à-dire qu'ils tâchent de récupérer la mémoire en réécrivant l'histoire pour mettre en lumière la façon dont l'État castillan - espagnol - a, au long de leur histoire commune, toujours fait preuve d'autoritarisme envers la Catalogne. On est là dans une historiographie qui, dans ses principes, rappellecelle de la Renaixença ${ }^{14}$, puisque le Cercle Català d'Història reprend presque mot pour mot le projet historiographique d'un Víctor Balaguer, auteur d'une importante histoire de la Catalogne dans les années $1860^{15}$. En revanche, Antoni Muñoz et Josep Catà soulèvent, par leur démarche, deux questions épistémologiques, à savoir celle du rapport à la narration dans la pratique de l'historien et celle du « point de vue».

\section{Point de vue et narration}

Il ne s'agit pas, ici, de revenir sur le débat portant sur «objectivité vs subjectivité » à propos duquel ont déjà écrit d'éminents historiens, à commencer par Marc Bloch, Michel de Certeau et Paul Veyne, pour n'en citer que quelques-uns ${ }^{16}$. Il ne fait aucun doute que tout projet historiographique est l'expression d'une subjectivité, et ce malgré le travail de distanciation. Quand bien même l'historien souhaiterait y échapper, le choix du sujet, celui des sources, l'opération historiographique et l'écriture même sont guidés par son triple engagement : personnel, social et institutionnel ${ }^{17}$.

11 Antoni Muñoz et Josep Catà déclarent, dès l'introduction et les premiers chapitres, vouloir construire un « récit» "non neutre» de ce qui s'est produit entre 1640 et $1641^{18}$. Ils offrent une narration argumentée et linéaire de la montée des tensions entre le gouvernement royal et les institutions catalanes jusqu'à la rupture de 1641. Ils choisissent délibérément de faire porter leurs efforts sur la politique vexatoire d'olivares plutôt que sur les tentatives d'apaisement auxquelles ont pourtant participé, certes en vain, les ambassadeurs de la Generalitat - sans doute plus que ceux du Consell de Cent qui avaient été assignés à résidence dès leur arrivée à Alcalá ${ }^{19}$. Leur propos consiste à se livrer à une lecture "alternative", selon leurs propres termes, de l'enchaînement des événements, en procédant à une sélection de documents d'archives et de citations (p.7-8) pour confronter le point de vue des victimes à celui des vainqueurs - ou plutôt des oppresseurs selon leur « point de vue»- qu'avaient restitué 
des historiens comme John H. Elliott, Josep Sanabre ou encore Eulogio Zudaire (p. 7-8). La Catalogne et les "patriotes » (p. 146) se trouvent ainsi installés, au sens canonique du terme, en victimes de la catalanophobie madrilène (p. 192).

Le choix du mode narratif plonge en outre le lecteur dans une succession linéaire de décisions «cyniques» (p. 135) du Comte-Duc et de ses ministres qui éprouvaient une «authentique délectation» (p. 186) à l'idée d'envahir militairement la Catalogne. Les excès des soldats sont donc décrits comme le déploiement d'une politique programmée de répression (p. 175) et d'une "solution finale» (p. 153). Il est renforcé par tout un système d'adjectivation - "pauvres » Catalans, "une décision profondément injuste " (p. 44-45) - par lequel les auteurs expriment leur "point de vue ", autrement dit, par lequel ils se posent en juges de l'histoire. Ce qui confortera le lecteur dans sa conviction que la Castille, totalitaire, centralisatrice et uniformisatrice, a envahi puis occupé la Catalogne à partir de 1640. Se pose dès lors la question de savoir comment l'historien doit réagir face à une telle approche ? Quelle(s) position(s) doit-il et peut-il adopter ?

Il peut, comme l'a plusieurs fois suggéré Henry Kamen, se contenter de «rétablir la vérité » et de dénoncer les falsifications du discours historique indépendantiste, d'en montrer les limites ${ }^{20}$. Mais cette méthode comporte des risques. Premièrement, elle place les différentes écoles historiographiques dans un dialogue symétrique, où plus aucun débat n'est possible. Deuxièmement, elle minimise les apports scientifiques que peuvent offrir les recherches menées par les historiens indépendantistes qui, malgré les différends historiographiques et idéologiques auxquels elles peuvent donner lieu, n'en restent pas moins des travaux scientifiques. Car l'ouvrage ici recensé apporte de nombreux renseignements, on l'a dit, sur la dimension sociale du mouvement paysan et sur le rôle central qu'ont joué les Comtés. Troisièmement, armer un contre-récit fait courir le risque de poser les jalons, volontairement ou non, d'un discours mémoriel concurrentiel. Quatrièmement, elle oblige à réduire considérablement le champ des recherches en concentrant les efforts sur des événements, des périodes et des problématiques extrêmement limitées, alors que les archives catalanes recèlent d'objets historiques à traiter ou à relire.

14 Mais la question du "point de vue » milite aussi pour une histoire plurielle, en lien, notamment, avec l'histoire des minorités nationales. Ou plutôt, pour reprendre le titre de l'ouvrage de Romain Bertrand, pour une «histoire à parts égales", autrement dit une histoire qui parviendrait à connecter les différents territoires de la Monarchie Catholique ${ }^{21}$. En Catalogne, le discours nationaliste, qui s'est consolidé dans les années 1990, a pour vocation d'offrir un discours historique destiné à proposer un discours historiographique "alternatif » - pour reprendre les termes de l'ouvrage recensé - à celui, plus traditionnel, centré autour de la Castille et de l'institution royale. La tâche de l'historien reviendrait donc à envisager cette pluralité des voix dans ses travaux pour en faire une synthèse, pour envisager d'autres pistes de réflexion, d'autres approches, d'autres outils ou, plus encore, pour définir de nouveaux objets d'études. Cette liste, bien entendu, ne se prétend pas exhaustive.

15 Cependant, le discours nationaliste doit aussi interpeller en tant qu'objet historique. Premièrement, parce qu'il doit être replacé au cœur des questions mémorielles qui secouent aujourd'hui l'ensemble de la société espagnole. Il questionne en effet les fondements du modèle d'État issu de la Transition ainsi que le rapport des citoyens à l'institution monarchique. Deuxièmement, il interroge la place des minorités nationales à la fois dans la Constitution et au sein d'un État jugé trop centralisateur, mais aussi 
dans la redéfinition des échelles et des régions survenue dans le processus de construction européenne. Enfin, il laisse entrevoir d'étroits liens, qui méritent d'être étudiés, avec les conséquences sociales de la crise économique des années 2000. En d'autres termes, il appartient aux historiens, modernistes et du temps présent, de se saisir de cette tonalité victimisante, tant regrettée et critiquée, pour analyser ce qu'elle a à nous dire de la société et de l'époque qui, simultanément, la produisent et la nourrissent.

\section{NOTES}

1. Pour qualifier cette méthode proposée par les auteurs, nous empruntons le terme " ralenti », déjà employé par Andreas Suter dans son article « Histoire sociale et événements historiques. Pour une nouvelle approche », Annales HSS, mai-juin 1997, no3, p. 543-567. Le français « ralenti » traduit l'allemand „Zeitlupe”, p. 544.

2. John H. Elliott, La rebelión de los catalanes (1598-1640) [1963], Madrid, Siglo XXI, 1977 ; Josep Sanabre, La Acción de Francia en Cataluña, Barcelone, Acadèmia de Bones Lletres de Barcelona, 1959 et Eulogio Zudaire, El Conde-duque y Cataluña, Madrid, CSIC, 1964.

3. Voir http://www.cch.cat/php/ls.php?fx=cat, consulté le 30 novembre 2017.

4. “[...] mostrar la vàlua i la preparació d'aquells homes [les ambassadeurs], a fi d'honorar-los com cal", p. 6. C'est nous qui traduisons les citations du catalan.

5. John H. Elliott, La rebelión de los catalanes (1598-1640), Madrid, Siglo XXI, 1977 et Josep Sanabre, La Acción de Francia en Cataluña, Barcelone, Reial Acadèmia de Bones Lletres, 1956.

6. Basilio de Rubí, Un segle de vida caputxina a Catalunya (1564-1664). Aproximació històricobibliogràfica, Barcelone, Favència, 1978 et Mathias Ledroit, « Les ambassadeurs catalans à Madrid en 1640 : chronique d'une guerre annoncée?», dans Béatrice Perez (dir.), Ambassadeurs, apprentis espions et maîtres comploteurs. Les systèmes de renseignement en Espagne à l'époque moderne, Paris, PUPS, 2010, p. 333-341.

7. Mathias Ledroit, Philippe IV et les Catalans, Paris, Presses Universitaires Paris-Sorbonne, coll. «Ibérica », à paraître.

8. Mathias Ledroit, «La légation de Francesc Bru, ambassadeur de Barcelone auprès de Philippe IV (juin-novembre 1632) », intervention à l'Université Paris-Sorbonne, dans le séminaire CLEA-CHECLA dirigé par Béatrice Perez, le 9 décembre 2017.

9. Pierre Vilar, La Catalogne dans l'Espagne moderne, Paris, S.E.V.P.E.N., 1962, 3 vol. Cette thèse de doctorat a été publiée en Espagne par Crítica en 1978 sous le titre de Cataluña en la España moderna. Elle a entraîné de nombreuses recherches, dans les années 1980, sur l'histoire économique et sociale de la Catalogne. On pensera, notamment, à la thèse d'Eva Serra, Pagesos $i$ senyors a la Catalunya del segle XVII. Baronia de Sentmenat, Barcelone, Crítica, 1988.

10. Nous renvoyons ici aux travaux d'Héloïse Hermant sur la révolte des Barratines.

11. Òscar Jané, Catalunya i França al segle XVII. Identitat, contraidentitat i ideologies a l'època moderna (1640-1700), Catarroja/Barcelone, Editorial Afers, 2006.

12. Eva Serra, "1640: una revolució política. La implicació de les institucions", dans Eva Serra (dir.), La Revolució catalana de 1640, Barcelone, Crítica, 1991, p. 3-65. Précisons que l'ouvrage recensé a été publié à l'occasion de la commémoration du $375^{\mathrm{e}}$ anniversaire de l'éclatement de la 
révolte de 1640, comme il est indiqué à la page 301. Cette commémoration, survenue dans la suite de celles de 1714, n'a bénéficié que de très peu d'écho médiatique.

13. Paul Ricœur, La mémoire, l'histoire, l'oubli, Paris, Édition du Seuil, 2000, p. 96-105.

14. Le mot Renaixença, dérivé du verbe « renàixer » - renaître - désigne la Renaissance catalane, un mouvement culturel amorcé dans la seconde moitié du XIX ${ }^{e}$ siècle, à l'époque de l'émergence des nationalismes européens. Il s'agissait de faire « renaître » la culture et la langue catalanes. On prendra soin de ne pas confondre la Renaixença avec le Renaixement, qui désigne la Renaissance du $\mathrm{XVI}^{\mathrm{e}}$ siècle.

15. Víctor Balaguer, Historia de Cataluña y de la Corona de Aragón, Barcelone, Salvador Moreno, 1863, 4. vol.

16. Marc Bloch, L'Étrange défaite [1940], Paris, Acalt Édition, 2016 ; Michel de Certeau, L'Écriture de l'histoire, Paris, Gallimard, coll. «Folio », 2002 et Paul Veyne, Comment on écrit l'histoire, Paris, Édition du Seuil, 1971.

17. Paul Ricœur, op. cit., p. 437.

18. Le mot « histoire », dans les langues latines, est particulièrement ambigu en ceci qu'il désigne tant la discipline historique que le récit. L'anglais, ici, est plus précis en distinguant history et story. Sans doute y aurait-il ici matière à creuser pour aborder l'historiographie nationaliste en tant qu'objet d'étude.

19. Mathias Ledroit, «Les ambassadeurs catalans à Madrid en 1640 : chronique d'une guerre annoncée? ", dans Béatrice Perez (dir.), Ambassadeurs, apprentis espions et maîtres comploteurs. Les systèmes de renseignement en Espagne à l'époque moderne, Paris, PUPS, 2010, p. 333-341

20. Henry Kamen, España y Cataluña. Historia de una pasión, Madrid, La Esfera de los libros, 2014. Traduction de l'anglais par José C. Vales.

21. Romain Bertrand, L'Histoire à parts égales, Paris, Seuil, 2011.

\section{AUTHORS}

\section{MATHIAS LEDROIT}

Université Paris Est Marne-la-Vallée, Laboratoire LISAA-EMHIS EA 4120. 